\title{
Poisson Quantum Information
}

\author{
Mankei Tsang ${ }^{1,2}$ \\ ${ }^{1}$ Department of Electrical and Computer Engineering, National University of Singapore, 4 Engineering Drive 3, Singapore \\ 117583 \\ ${ }^{2}$ Department of Physics, National University of Singapore, 2 Science Drive 3, Singapore 117551
}

By taking a Poisson limit for a sequence of rare quantum objects, I derive simple formulas for the Uhlmann fidelity, the quantum Chernoff quantity, the relative entropy, and the Helstrom information. I also present analogous formulas in classical information theory for a Poisson model. An operator called the intensity operator emerges as the central quantity in the formalism to describe Poisson states. It behaves like a density operator but is unnormalized. The formulas in terms of the intensity operators not only resemble the general formulas in terms of the density operators, but also coincide with some existing definitions of divergences between unnormalized positivesemidefinite matrices. Furthermore, I show that the effects of certain channels on Poisson states can be described by simple maps for the intensity operators.

\section{Introduction}

The Poisson limit theorems, also called the laws of rare events or the laws of small numbers, underlie the ubiquity of Poisson statistics and enable a variety of simplifications in probability theory $[1,2]$. In this paper, I examine the consequences of taking a similar limit in quantum information theory and demonstrate that elegant formulas emerge under the limit. This body of work may be called Poisson quantum information, which has the potential to grow into a fruitful research topic on par with Gaussian quantum information $[3,4]$.

The results put forth are a generalization and culmination of our earlier efforts concerning weak thermal light [5-8]. The results are especially relevant to the recent literature on the application of quantum information theory to partially coherent imaging [8-17], where there is some confusion regarding the correct formulas for the information quantities, leading to inconsistent results by different groups. This paper resolves the debate in support of Refs. [6, 10] and extends the results, beyond the Helstrom and Fisher information quantities considered there. While special weakthermal-light models have already found success by enabling substantial simplifications in many previous studies in quantum optics [5, 6, 8, 18-21], the general theory here stands on its own and does not require the quantum state to be bosonic or exactly thermal, so it may be applied to particles and systems beyond photons, such as electrons and quasiparticles, whenever a quantum treatment of rare objects is needed.

\section{Poisson states}

Let $\mathcal{P}(\mathcal{H})$ be the set of positive-semidefinite operators on a Hilbert space $\mathcal{H}$ and $\mathcal{P}_{1}(\mathcal{H})$ be the unit-trace subset $\mathcal{P}_{1}(\mathcal{H}) \equiv\{\tau \in \mathcal{P}(\mathcal{H}) \mid \operatorname{tr} \tau=1\}$. Consider the Hilbert space $\left(\mathcal{H}_{0} \oplus \mathcal{H}_{1}\right)^{\otimes M}$ for $M$ temporal modes, where $\mathcal{H}_{0}$ is a 1 -dimensional vacuum Hilbert space and $\mathcal{H}_{1}$ is a $d$-dimensional Hilbert space for a quantum object. Let the density operator $\rho_{M} \in \mathcal{P}_{1}\left[\left(\mathcal{H}_{0} \oplus \mathcal{H}_{1}\right)^{\otimes M}\right]$ be

$$
\rho_{M}=\tau^{\otimes M}, \quad \tau=\bigoplus_{l=0}^{1} \pi_{l} \tau_{l}, \quad \pi_{0}=1-\epsilon, \quad \pi_{1}=\epsilon,
$$

Mankei Tsang: mankei@nus.edu.sg, https://blog.nus.edu.sg/mankei/ 
where $\tau_{0} \in \mathcal{P}_{1}\left(\mathcal{H}_{0}\right)$ is the vacuum state, $\tau_{1} \in \mathcal{P}_{1}\left(\mathcal{H}_{1}\right)$ is the density operator for the quantum object, and $0 \leq \epsilon \leq 1$ is the probability of having one object in each temporal mode. See, for example, Ref. [22] for the definitions of the mathematical concepts. In the context of optics, $\tau_{1}$ may denote the one-photon density operator in $d$ spatial and polarization modes. The formalism, in itself, is generic however - the object can be any elementary or composite quantum system. For example, to study intensity interferometry [23], where two-photon-coincidence events are postselected and one-photon events are ignored, $\tau_{1}$ can be used to denote the two-photon density operator.

Define the Poisson limit as

$$
\epsilon \rightarrow 0, \quad M \rightarrow \infty, \quad N \equiv M \epsilon \quad \text { fixed, }
$$

where $N$ is the expected object number in total. In this limit, a central quantity in the ensuing theory is

$$
\Gamma \equiv N \tau_{1} \in \mathcal{P}\left(\mathcal{H}_{1}\right)
$$

which I call the intensity operator. Similar to $\tau_{1}$, it is a positive-semidefinite operator on $\mathcal{H}_{1}$. Unlike $\tau_{1}$, however, its trace

$$
\operatorname{tr} \Gamma=N
$$

is not normalized, as the object number need not be conserved in a problem.

Let $\mathcal{N}^{(k)} \equiv\left(\mathcal{N}_{1}^{(k)}, \mathcal{N}_{2}^{(k)}, \ldots\right)$ be the vectoral random variable from a multi-output objectcounting measurement of the $k$ th temporal mode. Let its probability distribution be

$$
\begin{aligned}
P_{\mathcal{N}^{(k)}}(0, \ldots, 0) & =\operatorname{tr} I_{0} \tau=1-\epsilon, \\
P_{\mathcal{N}^{(k)}}\left(0, \ldots, n_{j}=1, \ldots, 0\right) & =\operatorname{tr} E_{j} \tau=\epsilon \operatorname{tr} E_{j} \tau_{1},
\end{aligned}
$$

where $I_{l}$ is the projection operator into $\mathcal{H}_{l}$ and $E$ is a positive operator-valued measure (POVM) on $\mathcal{H}_{1}$ that satisfies $E_{j} \in \mathcal{P}\left(\mathcal{H}_{1}\right)$ and $\sum_{j} E_{j}=I_{1}$. Under the Poisson limit, the integrated random variable $\mathcal{M} \equiv \sum_{k=1}^{M} \mathcal{N}^{(k)}$ has the Poisson distribution [1, 24]

$$
P_{\mathcal{M}}(m)=\prod_{j} \exp \left(-\Lambda_{j}\right) \frac{\Lambda_{j}^{m_{j}}}{m_{j} !},
$$

where each intensity value is given by

$$
\Lambda_{j}=\operatorname{tr} E_{j} \Gamma .
$$

Appendix A states, in more rigorous terms, a Poisson limit theorem that gives Eqs. (2.7) and (2.8).

In view of the Poissonian properties, the quantum state given by Eqs. (2.1), together with the Poisson limit given by Eqs. (2.2), may be called a Poisson state, denoted as $\rho$ without the subscript $M$. Although a rigorous treatment of Poisson states may require quantum stochastic calculus [25] or nonstandard analysis [26, 27], in the following I take the more convenient approach of assuming Eqs. (2.1) and taking the Poisson limit at the end of a calculation. Appendix B discusses how Poisson states may be defined more rigorously in terms of Fock spaces.

\section{Information quantities}

To define information quantities, assume two Poisson states $\rho$ and $\rho^{\prime}$. Assume that $M$ and $\tau_{0}$ are the same for the two states, while the other quantities may vary. The quantities for the second state are denoted with a prime; for example, the intensity operator is $\Gamma^{\prime}$ and $N^{\prime} \equiv M \epsilon^{\prime}=\operatorname{tr} \Gamma^{\prime}$. Assume further that $\epsilon^{\prime}=O(\epsilon)$ (order at most $\epsilon$ in the Poisson limit), such that the Poisson limit applies to both states. My first proposition concerns the Uhlmann fidelity [28], the most notable application of which is to set useful bounds for quantum hypothesis testing [29]. 
Proposition 1. The Uhlmann fidelity

$$
F\left(\rho, \rho^{\prime}\right) \equiv \operatorname{tr} \sqrt{\sqrt{\rho} \rho^{\prime} \sqrt{\rho}}
$$

between two Poisson states is given by

$$
F\left(\rho, \rho^{\prime}\right)=\exp \left[-\frac{N+N^{\prime}}{2}+F\left(\Gamma, \Gamma^{\prime}\right)\right] .
$$

Proof. Given Eqs. (2.1) and using basic linear algebra (see, for example, Refs. [22, Chap. 1] and [25, Proposition 19.1]), it can be shown that

$$
F\left(\rho_{M}, \rho_{M}^{\prime}\right)=\left[F\left(\tau, \tau^{\prime}\right)\right]^{M}=\left[\sum_{l} F\left(\pi_{l} \tau_{l}, \pi_{l}^{\prime} \tau_{l}^{\prime}\right)\right]^{M}=\left[1-\frac{\epsilon+\epsilon^{\prime}}{2}+\sqrt{\epsilon \epsilon^{\prime}} F\left(\tau_{1}, \tau_{1}^{\prime}\right)+O\left(\epsilon^{2}\right)\right]^{M} .
$$

Taking the Poisson limit then leads to the proposition.

Equation (3.2) has a self-similar feature: it contains a fidelity expression, in terms of the intensity operators $\Gamma$ and $\Gamma^{\prime}$, that has the same form as the general formula for the density operators. Another remarkable feature is that the $d_{B}^{2}$ quantity, defined in the following expression

$$
-2 \ln F\left(\rho, \rho^{\prime}\right)=N+N^{\prime}-2 F\left(\Gamma, \Gamma^{\prime}\right) \equiv d_{B}^{2}\left(\Gamma, \Gamma^{\prime}\right),
$$

coincides with the squared Bures-Wasserstein distance between unnormalized positive-semidefinite operators $[28,30]$.

The next two propositions concern the quantum Chernoff quantity and the relative entropy. The Chernoff quantity is used in the quantum Chernoff bound for hypothesis testing [29, 31, 32], while the relative entropy is, of course, a fundamental quantity in quantum thermodynamics [33] and communication theory $[4,29]$.

Proposition 2. The quantum Chernoff quantity

$$
C_{s}\left(\rho, \rho^{\prime}\right) \equiv \operatorname{tr} \rho^{s} \rho^{\prime-s}, \quad 0 \leq s \leq 1
$$

for two Poisson states is given by

$$
C_{s}\left(\rho, \rho^{\prime}\right)=\exp \left[-s N-(1-s) N^{\prime}+C_{s}\left(\Gamma, \Gamma^{\prime}\right)\right] .
$$

The quantum Chernoff distance [31, 32] is then

$$
-\ln \left[\inf _{0 \leq s \leq 1} C_{s}\left(\rho, \rho^{\prime}\right)\right]=\sup _{0 \leq s \leq 1}\left[s N+(1-s) N^{\prime}-C_{s}\left(\Gamma, \Gamma^{\prime}\right)\right] .
$$

Proposition 3. The relative entropy

$$
D\left(\rho \| \rho^{\prime}\right) \equiv \operatorname{tr} \rho^{\prime}-\operatorname{tr} \rho+\operatorname{tr} \rho\left(\ln \rho-\ln \rho^{\prime}\right)
$$

between two Poisson states is given by

$$
D\left(\rho \| \rho^{\prime}\right)=D\left(\Gamma \| \Gamma^{\prime}\right) .
$$

If $\operatorname{supp} \Gamma \nsubseteq \operatorname{supp} \Gamma^{\prime}$, where supp denotes the support [4], then $\operatorname{supp} \rho \nsubseteq \operatorname{supp} \rho^{\prime}$, and $D\left(\rho \| \rho^{\prime}\right)=$ $D\left(\Gamma \mid \| \Gamma^{\prime}\right)=\infty$.

The proofs of Propositions 2 and 3 are delegated to Appendix C.

In Eq. (3.8), the relative entropy is expressed in a more general form so that it is appropriate for unnormalized positive-definite operators as well [34-36]. Similar to Eq. (3.2), Eqs. (3.6) and 
(3.9) have a self-similar feature. It is also remarkable that the $D_{s}\left(\Gamma, \Gamma^{\prime}\right)$ and $D\left(\Gamma \| \Gamma^{\prime}\right)$ quantities, defined in the following expressions

$$
\begin{aligned}
-\frac{1}{s(1-s)} \ln C_{s}\left(\rho, \rho^{\prime}\right) & =\frac{1}{s(1-s)}\left[s N+(1-s) N^{\prime}-C_{s}\left(\Gamma, \Gamma^{\prime}\right)\right] \equiv D_{s}\left(\Gamma, \Gamma^{\prime}\right), \\
D\left(\Gamma \| \Gamma^{\prime}\right) & \equiv N^{\prime}-N+\operatorname{tr} \Gamma\left(\ln \Gamma-\ln \Gamma^{\prime}\right),
\end{aligned}
$$

coincide with the alpha-divergences between unnormalized positive-semidefinite matrices in the matrix-analysis literature $[35,36]-D_{s}\left(\Gamma, \Gamma^{\prime}\right)$ here is identical to Eq. (4.179) in Ref. [36] if one sets $s=(1-\alpha) / 2$ and $D\left(\Gamma \| \Gamma^{\prime}\right)$ here is identical to Eq. (4.166) in Ref. [36].

The classical Poisson model given by Eq. (2.7) leads to analogous formulas for the corresponding quantities in classical information theory. The proofs are trivial and omitted for brevity; see, for example, Ref. [37] for similar results. Assume two Poisson distributions and again denote the quantities for the second distribution with a prime.

Proposition 4. The Chernoff quantity

$$
C_{s}\left(P_{\mathcal{M}}, P_{\mathcal{M}}^{\prime}\right) \equiv \sum_{m} P_{\mathcal{M}}^{s} P_{\mathcal{M}}^{\prime 1-s}, \quad 0 \leq s \leq 1
$$

for two Poisson distributions is given by

$$
C_{s}\left(P_{\mathcal{M}}, P_{\mathcal{M}}^{\prime}\right)=\exp \left[-s N-(1-s) N^{\prime}+C_{s}\left(\Lambda, \Lambda^{\prime}\right)\right]
$$

The Chernoff distance is then

$$
-\ln \left[\inf _{0 \leq s \leq 1} C_{s}\left(P_{\mathcal{M}}, P_{\mathcal{M}}^{\prime}\right)\right]=\sup _{0 \leq s \leq 1}\left[s N+(1-s) N^{\prime}-C_{s}\left(\Lambda, \Lambda^{\prime}\right)\right] .
$$

Proposition 5. The relative entropy

$$
D\left(P_{\mathcal{M}} \| P_{\mathcal{M}}^{\prime}\right) \equiv \sum_{m}\left(P_{\mathcal{M}}^{\prime}-P_{\mathcal{M}}+P_{\mathcal{M}} \ln \frac{P_{\mathcal{M}}}{P_{\mathcal{M}}^{\prime}}\right)
$$

between two Poisson distributions is given by

$$
D\left(P_{\mathcal{M}} \| P_{\mathcal{M}}^{\prime}\right)=D\left(\Lambda \| \Lambda^{\prime}\right)
$$

If $\operatorname{supp} \Lambda \nsubseteq \operatorname{supp} \Lambda^{\prime}$, then $\operatorname{supp} P_{\mathcal{M}} \nsubseteq \operatorname{supp} P_{\mathcal{M}}^{\prime}$, and $D\left(P_{\mathcal{M}} \| P_{\mathcal{M}}^{\prime}\right)=D\left(\Lambda \| \Lambda^{\prime}\right)=\infty$.

Equations (3.13) and (3.16) resemble Eqs. (3.6) and (3.9) and also possess a self-similar feature. Similar to the quantum case, the $D_{s}\left(\Lambda, \Lambda^{\prime}\right)$ and $D\left(\Lambda \| \Lambda^{\prime}\right)$ quantities, defined in the following expressions

$$
\begin{aligned}
-\frac{1}{s(1-s)} \ln C_{s}\left(P_{\mathcal{M}}, P_{\mathcal{M}}^{\prime}\right) & =\frac{1}{s(1-s)}\left[s N+(1-s) N^{\prime}-C_{s}\left(\Lambda, \Lambda^{\prime}\right)\right] \equiv D_{s}\left(\Lambda, \Lambda^{\prime}\right), \\
D\left(\Lambda \| \Lambda^{\prime}\right) & \equiv N^{\prime}-N+\sum_{j} \Lambda_{j} \ln \frac{\Lambda_{j}}{\Lambda_{j}^{\prime}}
\end{aligned}
$$

coincide with the alpha-divergences between unnormalized positive distributions [38]. $D_{1 / 2}\left(\Lambda, \Lambda^{\prime}\right) / 2$, in particular, is the squared Hellinger distance [30].

A fundamental relation between the quantum and classical information quantities is monotonicity. It is interesting to note that it manifests for the Poisson states on two levels: on the level of $\rho$ and on the level of $\Gamma$.

\section{Proposition 6.}

$$
\begin{aligned}
F\left(\rho, \rho^{\prime}\right) & \leq C_{1 / 2}\left(P_{\mathcal{M}}, P_{\mathcal{M}}^{\prime}\right), \\
C_{s}\left(\rho, \rho^{\prime}\right) & \leq C_{s}\left(P_{\mathcal{M}}, P_{\mathcal{M}}^{\prime}\right), \\
D\left(\rho \| \rho^{\prime}\right) & \geq D\left(P_{\mathcal{M}} \| P_{\mathcal{M}}^{\prime}\right) .
\end{aligned}
$$


Proof. These bounds follow directly from the monotonicity relations [29]. Alternatively, one may take $\Gamma=N \tau_{1}$ and $\Gamma^{\prime}=N^{\prime} \tau_{1}^{\prime}$, define $p_{j} \equiv \operatorname{tr} E_{j} \tau_{1}=\Lambda_{j} / N$ and $p_{j}^{\prime} \equiv \operatorname{tr} E_{j} \tau_{1}^{\prime}=\Lambda_{j}^{\prime} / N^{\prime}$, and apply the monotonicity relations with respect to $\left(\tau_{1}, \tau_{1}^{\prime}\right)$ and $\left(p, p^{\prime}\right)$ in order to obtain

$$
\begin{aligned}
F\left(\Gamma, \Gamma^{\prime}\right) & =\sqrt{N N^{\prime}} F\left(\tau_{1}, \tau_{1}^{\prime}\right) \leq \sqrt{N N^{\prime}} C_{1 / 2}\left(p, p^{\prime}\right)=C_{1 / 2}\left(\Lambda, \Lambda^{\prime}\right), \\
C_{s}\left(\Gamma, \Gamma^{\prime}\right) & =N^{s} N^{\prime 1-s} C_{s}\left(\tau_{1}, \tau_{1}^{\prime}\right) \leq N^{s} N^{\prime 1-s} C_{s}\left(p, p^{\prime}\right)=C_{s}\left(\Lambda, \Lambda^{\prime}\right), \\
D\left(\Gamma \| \Gamma^{\prime}\right) & =N^{\prime}-N+N \ln \frac{N}{N^{\prime}}+N D\left(\tau_{1} \| \tau_{1}^{\prime}\right) \geq N^{\prime}-N+N \ln \frac{N}{N^{\prime}}+N D\left(p \| p^{\prime}\right)=D\left(\Lambda \| \Lambda^{\prime}\right),
\end{aligned}
$$

which also lead to the bounds via Propositions 1-5.

Last but not the least, I present propositions concerning the Helstrom information and the Fisher information $[18,29]$, which play crucial roles in parameter estimation. Let $\epsilon$ and $\tau_{1}$ be functions of an unknown vectoral parameter $\theta \equiv\left(\theta_{1}, \ldots, \theta_{q}\right) \in \Theta \subseteq \mathbb{R}^{q}$. It follows that $\rho_{M}$ and $\Gamma$ are also functions of $\theta$.

Proposition 7. Define the $q \times q$ Helstrom information matrix as

$$
K_{\mu \nu}\left(\rho_{M}\right) \equiv \operatorname{tr}\left(\sigma_{\mu} \circ \sigma_{\nu}\right) \rho_{M},
$$

where $a \circ b \equiv(a b+b a) / 2$ denotes the Jordan product and $\sigma_{\mu}$ is a symmetric logarthmic derivative (SLD) of $\rho_{M}$, defined as a Hermitian-operator solution to

$$
\frac{\partial \rho_{M}}{\partial \theta_{\mu}}=\sigma_{\mu} \circ \rho_{M}
$$

For a Poisson state, $K$ is given by

$$
K_{\mu \nu}(\rho)=K_{\mu \nu}(\Gamma)=\operatorname{tr}\left(S_{\mu} \circ S_{\nu}\right) \Gamma,
$$

where $S_{\mu}$ is an $S L D$ of $\Gamma$, viz., a Hermitian-operator solution to

$$
\frac{\partial \Gamma}{\partial \theta_{\mu}}=S_{\mu} \circ \Gamma
$$

Proof. Given the tensor product in Eqs. (2.1), it is known that [29]

$$
K\left(\rho_{M}\right)=M K(\tau) .
$$

Given the direct sum in Eqs. (2.1), it can also be shown that

$$
K_{\mu \nu}(\tau)=\sum_{l} \operatorname{tr}\left(S_{\mu}^{(l)} \circ S_{\nu}^{(l)}\right) \pi_{l} \tau_{l},
$$

where $S_{\mu}^{(l)}$ is an SLD of $\pi_{l} \tau_{l}$. Then $S_{\mu}^{(0)}=\left(\partial \pi_{0} / \partial \theta_{\mu}\right) / \pi_{0}=-\left(\partial \epsilon / \partial \theta_{\mu}\right) /(1-\epsilon), S_{\mu}^{(1)}=S_{\mu}$, and

$$
K_{\mu \nu}\left(\rho_{M}\right)=\frac{1}{M(1-\epsilon)} \frac{\partial N}{\partial \theta_{\mu}} \frac{\partial N}{\partial \theta_{\nu}}+\operatorname{tr}\left(S_{\mu} \circ S_{\nu}\right) \Gamma .
$$

Taking the Poisson limit leads to the proposition.

Proposition 8 (well known; see, for example, Refs. [1, 2]). The Fisher information matrix

$$
J_{\mu \nu}\left(P_{\mathcal{M}}\right) \equiv \sum_{m} P_{\mathcal{M}} \frac{\partial \ln P_{\mathcal{M}}}{\partial \theta_{\mu}} \frac{\partial \ln P_{\mathcal{M}}}{\partial \theta_{\nu}}
$$

for a Poisson distribution is given by

$$
J\left(P_{\mathcal{M}}\right)=J(\Lambda)
$$


Again, with the Poisson limit, the Helstrom and Fisher information quantities observe a selfsimilar feature - they are given by the same formulas as the general ones, except that the unnormalized $\Gamma$ or $\Lambda$ is substituted into each.

The information quantities obey a monotonicity relation, which follows from the monotonicity of Helstrom information in two ways.

\section{Proposition 9.}

$$
K(\rho)=K(\Gamma) \geq J(\Lambda)=J\left(P_{\mathcal{M}}\right)
$$

in the sense that $K(\rho)-J\left(P_{\mathcal{M}}\right)$ and $K(\Gamma)-J(\Lambda)$ are positive-semidefinite.

Proof. The monotonicity [29] holds on two levels: $K(\rho) \geq J\left(P_{\mathcal{M}}\right)$, and also $K(\Gamma) \geq J(\Lambda)$, which can be proved independently by expressing $\Gamma$ and $\Lambda$ in terms of $N, \tau_{1}$, and $p=\Lambda / N$ and applying the monotonicity relation with respect to $\tau_{1}$ and $p$.

\section{Poisson states versus Fock states}

It is important to emphasize that the Poisson theory is in general different from the usual theory for the Fock state $\tau_{1}^{\otimes L}$. For example, from Eq. (3.2), the fidelity for the Poisson states can be expressed in terms of $\tau_{1}$ and $\tau_{1}^{\prime}$ as

$$
F\left(\rho, \rho^{\prime}\right)=\exp \left[-\frac{N+N^{\prime}}{2}+\sqrt{N N^{\prime}} F\left(\tau_{1}, \tau_{1}^{\prime}\right)\right],
$$

which is quite different from

$$
F\left(\tau_{1}^{\otimes L}, \tau_{1}^{\prime \otimes L}\right)=\left[F\left(\tau_{1}, \tau_{1}^{\prime}\right)\right]^{L}
$$

As $\Gamma$ and $\Gamma^{\prime}$ are not normalized, their fidelity is bounded as

$$
0 \leq F\left(\Gamma, \Gamma^{\prime}\right)=\sqrt{N N^{\prime}} F\left(\tau_{1}, \tau_{1}^{\prime}\right) \leq \sqrt{N N^{\prime}} .
$$

When $F\left(\tau_{1}, \tau_{1}^{\prime}\right)=1$ and $F\left(\Gamma, \Gamma^{\prime}\right)=\sqrt{N N^{\prime}}, F\left(\rho, \rho^{\prime}\right)$ in the Poisson theory is still less than 1 if $N \neq N^{\prime}$, because the different expected object numbers still lead to distinguishability. On the other hand, if $F\left(\tau_{1}, \tau_{1}^{\prime}\right)=0$ and $F\left(\Gamma, \Gamma^{\prime}\right)=0, F\left(\rho, \rho^{\prime}\right)$ is still positive, because both states contain the identical $\tau_{0}$. Another example is the Helstrom information

$$
K_{\mu \nu}(\rho)=K_{\mu \nu}(\Gamma)=N \frac{\partial \ln N}{\partial \theta_{\mu}} \frac{\partial \ln N}{\partial \theta_{\nu}}+N K_{\mu \nu}\left(\tau_{1}\right)
$$

which is different from

$$
K\left(\tau_{1}^{\otimes L}\right)=L K\left(\tau_{1}\right)
$$

It is not difficult to prove that, on the per-object basis,

$$
\frac{K(\Gamma)}{N} \geq K\left(\tau_{1}\right)
$$

as $N$ may also depend on $\theta$ and the total object number may give extra information.

In the context of optics, the thermal state in an ultraviolet limit can be shown to approach the Poisson state, with the mutual coherence matrix in statistical optics [23] becoming a matrix representation of $\Gamma[5,6,8]$. Alternative analyses of the exact thermal state have yielded results that are consistent with the Poisson theory [19, 39-41]. In the context of partially coherent imaging, Propositions 7 and 8 are consistent with our treatment in Ref. [10], although many other studies on this topic $[9,11-13,15]$ compute the Helstrom information using $K\left(\tau_{1}\right)$ only and may underestimate the amount of information. The Poisson model is more realistic than the Fock model because the former can account for the effects of inefficiency and loss, which are unavoidable for sources imaged with a finite aperture [17]. The use of $K\left(\tau_{1}\right)$ is justified only if $N$ does not depend on $\theta$, in which case $K(\Gamma)=N K\left(\tau_{1}\right)$. I illustrate these concepts with a concrete example. 
Example 1. Consider the imaging of two equally bright partially coherent optical sources via a diffraction-limited system in one dimension [8-17]. The intensity operator can be modeled as [10]

$$
\begin{aligned}
\Gamma & =N_{0}\left(\left|\psi_{1}\right\rangle\left\langle\psi_{1}|+| \psi_{2}\right\rangle\left\langle\psi_{2}|+\gamma| \psi_{1}\right\rangle\left\langle\psi_{2}\left|+\gamma^{*}\right| \psi_{2}\right\rangle\left\langle\psi_{1}\right|\right), \\
\left|\psi_{1}\right\rangle & =\int_{-\infty}^{\infty} d x \psi\left(x+\frac{\theta}{2}\right)|x\rangle, \quad\left|\psi_{2}\right\rangle=\int_{-\infty}^{\infty} d x \psi\left(x-\frac{\theta}{2}\right)|x\rangle,
\end{aligned}
$$

where $N_{0} \in \mathbb{R}_{>0} \equiv\{x \in \mathbb{R} \mid x \geq 0\}$ is the expected photon number from one isolated source, $\gamma \in \mathbb{C}$ is the degree of coherence with $|\gamma| \leq 1, \psi: \mathbb{R} \rightarrow \mathbb{C}$ is the point-spread function of the imaging system, $|x\rangle$ is the Dirac position eigenket that obeys $\left\langle x \mid x^{\prime}\right\rangle=\delta\left(x-x^{\prime}\right)$, and $\theta \in \mathbb{R}_{\geq 0}$ is the separation between the two sources in Airy units. The expected total photon number is

$$
N=\operatorname{tr} \Gamma=2 N_{0}\left[1+\operatorname{Re}\left(\gamma\left\langle\psi_{2} \mid \psi_{1}\right\rangle\right)\right] .
$$

When $\gamma=0$ (incoherent sources), $N=2 N_{0}$ is independent of $\theta$, but when $\gamma \neq 0, N$ may depend on $\theta$, as the waves from the two sources can interfere, thus enhancing or suppressing the radiation energy. For example, when $\gamma=1$ (fully coherent and in-phase sources) and $\theta=0, N=4 N_{0}$, which is consistent with the elementary fact that $n$ identical in-phase sources with zero separations should radiate at a power $\propto n^{2}$ [42].

To compute the Helstrom information $K(\Gamma)$, I assume the Gaussian point-spread function

$$
\psi(x)=\frac{1}{(2 \pi)^{1 / 4}} \exp \left(-\frac{x^{2}}{4}\right)
$$

and use the steps detailed in Appendix D to compute Eqs. (3.27) and (3.28) numerically. The results are plotted in Fig. 1. Almost identical results are recently reported in Ref. [17, Fig. 2(a)], which uses the rare-photon model given by Eqs. (2.1) and a theory consistent with the one here. The use of the intensity operator here is arguably more direct and convenient, however, as Eq. (4.7) comes naturally from optics and can be used directly in Eqs. (3.27) and (3.28), without returning to the rare-photon model or treating $\epsilon$ and $\tau_{1}$ separately in the calculations.

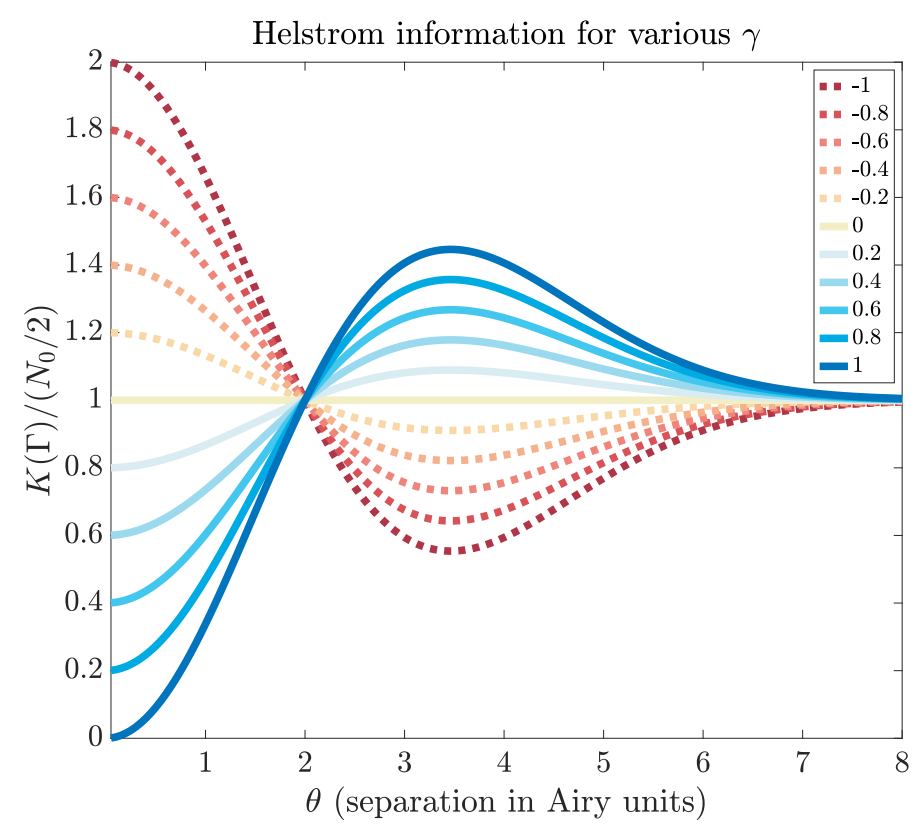

Figure 1: Numerically computed Helstrom information $K(\Gamma)$ for the estimation of the separation $\theta$ between two partially coherent sources. Each curve assumes a fixed degree of coherence $\gamma$, as denoted by the legend.

As also noticed in Ref. [17], the Helstrom information plotted in Fig. 1 appears to be identical to the Fisher information with the Hermite-Gaussian measurements plotted in Ref. [10, Fig. 1] for all values of $\gamma$, suggesting that the measurements are optimal for any $\gamma$ and not just for the $\gamma=0$ case proven in Ref. [6]. 


\section{Poisson channels}

For the Poisson theory to remain useful for problems involving quantum channels, the channels should preserve the Poissonianity of a state. I call such channels Poisson channels. A tracepreserving completely positive (TPCP) map $\Phi$ on $\rho_{M}$ is Poisson if

$$
\Phi\left(\rho_{M}\right)=\left[\left(1-\epsilon^{\prime \prime}\right) \tau_{0}^{\prime \prime} \oplus \epsilon^{\prime \prime} \tau_{1}^{\prime \prime}\right]^{\otimes M},
$$

where $\tau_{l}^{\prime \prime} \in \mathcal{P}_{1}\left(\mathcal{H}_{l}^{\prime \prime}\right), \mathcal{H}_{0}^{\prime \prime}$ is 1 -dimensional, and $\epsilon^{\prime \prime}=O(\epsilon)$, such that the output is still a Poisson state in the Poisson limit. Let $\rho(\Gamma)$ denote the Poisson state with intensity operator $\Gamma$ in the sense of Eqs. (2.1)-(2.3). The input-output relation in the Poisson limit can be abbreviated as

$$
\Phi[\rho(\Gamma)]=\rho[\tilde{\Phi}(\Gamma)], \quad \tilde{\Phi}(\Gamma)=N^{\prime \prime} \tau_{1}^{\prime \prime}, \quad N^{\prime \prime} \equiv M \epsilon^{\prime \prime},
$$

where $\tilde{\Phi}: \mathcal{P}\left(\mathcal{H}_{1}\right) \rightarrow \mathcal{P}\left(\mathcal{H}_{1}^{\prime \prime}\right)$ is a map from an intensity operator to another intensity operator induced by $\Phi$. Then the information quantities $d_{B}\left(\Gamma, \Gamma^{\prime}\right), D_{s}\left(\Gamma, \Gamma^{\prime}\right), D\left(\Gamma \| \Gamma^{\prime}\right)$, and $K(\Gamma)$ in Eqs. (3.4), (3.10), (3.11), and (3.27) also observe monotonicity relations with respect to $\tilde{\Phi}$.

Proposition 10. Let $\tilde{\Phi}$ be a positive map defined in the sense of Eqs. (5.1) and (5.2) with respect to a TPCP map $\Phi$ in the Poisson limit. Then

$$
\begin{aligned}
d_{B}\left(\Gamma, \Gamma^{\prime}\right) & \geq d_{B}\left[\tilde{\Phi}(\Gamma), \tilde{\Phi}\left(\Gamma^{\prime}\right)\right] \\
D_{s}\left(\Gamma, \Gamma^{\prime}\right) & \geq D_{s}\left[\tilde{\Phi}(\Gamma), \tilde{\Phi}\left(\Gamma^{\prime}\right)\right] \\
D\left(\Gamma \| \Gamma^{\prime}\right) & \geq D\left[\tilde{\Phi}(\Gamma) \| \tilde{\Phi}\left(\Gamma^{\prime}\right)\right], \\
K(\Gamma) & \geq K[\tilde{\Phi}(\Gamma)] . \quad(\text { if } \tilde{\Phi} \text { does not depend on } \theta)
\end{aligned}
$$

Proof. These monotonicity relations follow from the monotonicity of the information quantities with respect to the density operators in Eqs. (3.4), (3.10), (3.11), and (3.27).

To construct examples of $\tilde{\Phi}$ in the following, I assume further that $\Phi$ is local, in the sense of

$$
\Phi\left(\rho_{M}\right)=[\phi(\tau)]^{\otimes M}
$$

where $\phi: \mathcal{P}\left(\mathcal{H}_{0} \oplus \mathcal{H}_{1}\right) \rightarrow \mathcal{P}\left(\mathcal{H}_{0}^{\prime \prime} \oplus \mathcal{H}_{1}^{\prime \prime}\right)$ is a TPCP map that gives

$$
\phi(\tau)=\left(1-\epsilon^{\prime \prime}\right) \tau_{0}^{\prime \prime} \oplus \epsilon^{\prime \prime} \tau_{1}^{\prime \prime} .
$$

Under the local assumption, $\tilde{\Phi}$ is affine, as shown by Proposition 11 below, although Example 3 later demonstrates that $\tilde{\Phi}$ need not be trace-preserving and Example 4 demonstrates that $\tilde{\Phi}$ need not be linear.

Proposition 11. Given Eqs. (5.7) and (5.8), $\tilde{\Phi}$ is affine.

Proof. Let the Kraus form [22, 29] of $\phi$ be

$$
\phi(\tau)=\sum_{\alpha} A_{\alpha} \tau A_{\alpha}^{\dagger}
$$

Expressing $\tau$ and $A_{\alpha}$ in the matrix forms [22, Eq. (1.64)]

$$
\tau=\left(\begin{array}{cc}
(1-\epsilon) \tau_{0} & 0 \\
0 & \epsilon \tau_{1}
\end{array}\right), \quad A_{\alpha}=\left(\begin{array}{cc}
A_{\alpha 00} & A_{\alpha 01} \\
A_{\alpha 10} & A_{\alpha 11}
\end{array}\right)
$$

where 0 denotes a zero operator and $A_{\alpha l m}$ is an operator that maps $\mathcal{H}_{m}$ to $\mathcal{H}_{l}^{\prime \prime}$, it can be shown that

$$
\epsilon^{\prime \prime} \tau_{1}^{\prime \prime}=\sum_{\alpha}\left[A_{\alpha 10}(1-\epsilon) \tau_{0} A_{\alpha 10}^{\dagger}+A_{\alpha 11} \epsilon \tau_{1} A_{\alpha 11}^{\dagger}\right]
$$


For the $\epsilon^{\prime \prime}=O(\epsilon)$ requirement to be satisfied, it is necessary for the map to satisfy

$$
\begin{aligned}
& \operatorname{tr} \sum_{\alpha} A_{\alpha 10} \tau_{0} A_{\alpha 10}^{\dagger}=O(\epsilon), \\
& \operatorname{tr} \sum_{\alpha} A_{\alpha 11} \tau_{1} A_{\alpha 11}^{\dagger}=O(1) .
\end{aligned}
$$

Multiplying both sides of Eq. (5.11) by $M$ and taking the Poisson limit, the output intensity operator becomes

$$
\tilde{\Phi}(\Gamma)=\Gamma^{\prime}+\sum_{\alpha} A_{\alpha 11} \Gamma A_{\alpha 11}^{\dagger}
$$

where $\Gamma^{\prime}$ is the Poisson limit of $M(1-\epsilon) \sum_{\alpha} A_{\alpha 10} \tau_{0} A_{\alpha 10}^{\dagger} \rightarrow M \sum_{\alpha} A_{\alpha 10} \tau_{0} A_{\alpha 10}^{\dagger}$, which does not depend on $\Gamma$. Equation (5.14) is affine with respect to $\Gamma$ (though not necessarily linear).

In the following examples, the Kraus form of each $\phi$ is most conveniently expressed in terms of some orthonormal bases of $\mathcal{H}_{0}, \mathcal{H}_{0}^{\prime \prime}, \mathcal{H}_{1}$, and $\mathcal{H}_{1}^{\prime \prime}$, which are written as $\{|0\rangle\},\left\{\left|0^{\prime \prime}\right\rangle\right\},\left\{\left|1_{j}\right\rangle \mid j=\right.$ $1, \ldots, d\}$, and $\left\{\left|1_{k}^{\prime \prime}\right\rangle \mid k=1, \ldots, d^{\prime \prime}\right\}$, respectively. The $\tau$ state, for example, can be expressed as

$$
(1-\epsilon) \tau_{0} \oplus \epsilon \tau_{1}=(1-\epsilon)|0\rangle\left\langle 0\left|+\epsilon \sum_{j, k} g_{j k}\right| 1_{j}\right\rangle\left\langle 1_{k}\right|,
$$

where $g$ is the density matrix of $\tau_{1}$ with respect to the basis above.

Example 2 (object-number-preserving channel). Let a Kraus form of $\phi$ be

$$
\begin{aligned}
\phi(\tau) & =A_{0} \tau A_{0}^{\dagger}+\sum_{\alpha} A_{\alpha} \tau A_{\alpha}^{\dagger}, \\
A_{0} & =\left|0^{\prime \prime}\right\rangle\left\langle 0\left|, \quad A_{\alpha}=\sum_{j, k} A_{\alpha j k}\right| 1_{j}^{\prime \prime}\right\rangle\left\langle 1_{k}\right|,
\end{aligned}
$$

such that

$$
\phi\left[(1-\epsilon) \tau_{0} \oplus \epsilon \tau_{1}\right]=(1-\epsilon) \tau_{0}^{\prime \prime} \oplus \epsilon \phi_{1}\left(\tau_{1}\right)
$$

where

$$
\phi_{1}\left(\tau_{1}\right)=\sum_{\alpha} A_{\alpha} \tau_{1} A_{\alpha}^{\dagger}
$$

is a TPCP map. In the Poisson limit,

$$
\tilde{\Phi}(\Gamma)=\phi_{1}(\Gamma)
$$

The trace-preserving nature of $\phi_{1}$ means that the channel preserves the object number. More specific examples include the unitary map

$$
\phi_{1}(\Gamma)=U \Gamma U^{\dagger}
$$

which models a lossless linear device via a unitary operator $U$, and the map

$$
\phi_{1}(\Gamma)=\Lambda, \quad \Lambda_{j}=\operatorname{tr} E_{j} \Gamma,
$$

which models a 100\%-efficient object-counting measurement via the POVM $E$ that gives Eqs. (2.7) and (2.8). Propositions 6 and 9 are hence special cases of Proposition 10.

As the object number need not be conserved, $\tilde{\Phi}$ need not be trace-preserving. The next examples of $\tilde{\Phi}$ are noteworthy departures from the usual TPCP maps in quantum information theory. 
Example 3 (loss). Let $\mathcal{H}_{l}^{\prime \prime}=\mathcal{H}_{l}$ and

$$
\begin{aligned}
\phi(\tau) & =I_{0} \tau I_{0}+\sum_{j=1}^{d} A_{j} \tau A_{j}^{\dagger}+T \tau T, \\
I_{0} & =|0\rangle\left\langle 0\left|, \quad A_{j}=\sqrt{1-\eta_{j}}\right| 0\right\rangle\left\langle 1_{j}\left|, \quad T=\sum_{j=1}^{d} \sqrt{\eta_{j}}\right| 1_{j}\right\rangle\left\langle 1_{j}\right|,
\end{aligned}
$$

where $0 \leq \eta_{j} \leq 1$ is the transmission coefficient for each mode. Then

$$
\phi\left[(1-\epsilon) \tau_{0} \oplus \epsilon \tau_{1}\right]=\left(1-\epsilon \operatorname{tr} T \tau_{1} T\right) \tau_{0} \oplus \epsilon T \tau_{1} T
$$

In the Poisson limit,

$$
\tilde{\Phi}(\Gamma)=T \Gamma T
$$

This $\tilde{\Phi}$ is not trace-preserving if any $\eta_{j}<1$, although it is still completely positive [22, Theorem 2.22].

Example 4 (spontaneous emission). Let $\mathcal{H}_{l}^{\prime \prime}=\mathcal{H}_{l}$ and

$$
\begin{aligned}
\phi(\tau) & =A_{0} \tau A_{0}^{\dagger}+\sum_{j=1}^{d} A_{j} \tau A_{j}^{\dagger}+I_{1} \tau I_{1}, \\
A_{0} & =\sqrt{1-\epsilon^{\prime}}|0\rangle\left\langle 0\left|, \quad A_{j}=\sqrt{\epsilon^{\prime} \tau_{1 j j}^{\prime}}\right| 1_{j}\right\rangle\langle 0|, \\
I_{1} & =\sum_{j=1}^{d}\left|1_{j}\right\rangle\left\langle 1_{j}\right|,
\end{aligned}
$$

where $\epsilon^{\prime}=O(\epsilon)$ and $\tau_{1}^{\prime}=\sum_{j} \tau_{1 j j}^{\prime}\left|1_{j}\right\rangle\left\langle 1_{j}\right| \in \mathcal{P}_{1}\left(\mathcal{H}_{1}\right)$ are properties of the channel and $\left\{\left|1_{j}\right\rangle\right\}$ is assumed to be the eigenbasis of $\tau_{1}^{\prime}$ without loss of generality. Then

$$
\phi\left[(1-\epsilon) \tau_{0} \oplus \epsilon \tau_{1}\right]=(1-\epsilon)\left(1-\epsilon^{\prime}\right) \tau_{0} \oplus\left[\epsilon \tau_{1}+\epsilon^{\prime}(1-\epsilon) \tau_{1}^{\prime}\right]
$$

In the Poisson limit,

$$
\tilde{\Phi}(\Gamma)=\Gamma+\Gamma^{\prime}
$$

where $\Gamma^{\prime}=M \epsilon^{\prime} \tau_{1}^{\prime}$. In particular, if the input intensity operator is the zero operator 0 ,

$$
\tilde{\Phi}(0)=\Gamma^{\prime}
$$

If $\Gamma^{\prime} \neq 0, E q$. (5.32) implies that this $\tilde{\Phi}$ is not trace-preserving and not even linear, as a tracepreserving or linear map on the zero operator must give the zero operator.

Setting $\epsilon^{\prime}$, the spontaneous-emission probability per temporal mode, to be $O(\epsilon)$ ensures that the occurrence of objects remains rare and $N^{\prime \prime}=N+N^{\prime}$ remains finite under the Poisson limit. In reality, however, a channel with a high spontaneous-emission probability may make the final state non-Poisson and may also involve other processes such as stimulated emission, so one should double-check the accuracy of the Poisson approximation before applying the Poisson theory to a real spontaneous-emission channel.

Equation (5.31) is a quantum analog of the fact that the sum of two independent Poisson processes is also a Poisson process. It may be useful for modeling dark counts or background noise [43-45]. Another version of this fact is the following.

Example 5 (composition). The tensor product of two Poisson states may be expressed as

$$
\rho(\Gamma) \otimes \rho\left(\Gamma^{\prime}\right)=\rho\left(\Gamma \oplus \Gamma^{\prime}\right) .
$$


To derive this relation, consider

$$
\tau \otimes \tau^{\prime}=\left[(1-\epsilon)\left(1-\epsilon^{\prime}\right) \tau_{0} \otimes \tau_{0}^{\prime}\right] \oplus\left[\epsilon\left(1-\epsilon^{\prime}\right) \tau_{1} \otimes \tau_{0}^{\prime}\right] \oplus\left[\epsilon^{\prime}(1-\epsilon) \tau_{0} \otimes \tau_{1}^{\prime}\right] \oplus\left(\epsilon \epsilon^{\prime} \tau_{1} \otimes \tau_{1}^{\prime}\right) .
$$

Ignore all $O\left(\epsilon^{2}\right)$ terms in Eq. (5.34), including the two-object component $\epsilon \epsilon^{\prime} \tau_{1} \otimes \tau_{1}^{\prime}$. The output intensity operator in the Poisson limit becomes

$$
\left(\Gamma \otimes \tau_{0}^{\prime}\right) \oplus\left(\tau_{0} \otimes \Gamma^{\prime}\right) .
$$

Since $\tau_{0}=|0\rangle\langle 0|$ and $\tau_{0}^{\prime}=\left|0^{\prime}\right\rangle\left\langle 0^{\prime}\right|$ are 1-dimensional, any tensor product of an operator with $\tau_{0}$ or $\tau_{0}^{\prime}$ is isomorphic to the original operator, and Eq. (5.35) can be abbreviated as $\Gamma \oplus \Gamma^{\prime}$.

Example 6 (marginalization). Let $\Gamma$ be an intensity operator on $\mathcal{H}_{1} \oplus \mathcal{H}_{1}^{\prime}$ and $\tau$ be a density operator on $\left(\mathcal{H}_{0} \oplus \mathcal{H}_{1}\right) \otimes\left(\mathcal{H}_{0}^{\prime} \oplus \mathcal{H}_{1}^{\prime}\right)$. Let

$$
\phi(\tau)=\operatorname{tr}^{\prime} \tau
$$

denote the partial trace with respect to $\mathcal{H}_{0}^{\prime} \oplus \mathcal{H}_{1}^{\prime}$. A Kraus form is

$$
\phi(\tau)=\left\langle 0^{\prime}|\tau| 0^{\prime}\right\rangle+\sum_{j=1}^{d^{\prime}}\left\langle 1_{j}^{\prime}|\tau| 1_{j}^{\prime}\right\rangle .
$$

Expressing $\tau$ in terms of the basis $\left\{|0\rangle \otimes\left|0^{\prime}\right\rangle,\left|1_{j}\right\rangle \otimes\left|0^{\prime}\right\rangle,|0\rangle \otimes\left|1_{k}^{\prime}\right\rangle \mid j=1, \ldots, d, k=1, \ldots, d^{\prime}\right\}$, it is not difficult to show that

$$
\tilde{\Phi}(\Gamma)=I_{1} \Gamma I_{1},
$$

where $I_{1}$ is the projection operator into $\mathcal{H}_{1}$ given by Eq. (5.29).

Equations (5.20), (5.26), (5.31), (5.33), and (5.38) in the examples demonstrate that, for Poisson channels, the maps can be much simplified if expressed in terms of the intensity operators. Whether there exists a more comprehensive mathematical treatment of Poisson channels in the spirit of quantum channel theory is an interesting open problem.

\section{Conclusion}

In conclusion, I have shown that the Poisson limit leads to elegant results in quantum information theory, with the intensity operator emerging as the central quantity. The familiar appearances of the formulas mean that one may borrow existing results from general information theory and apply them to the intensity operators in the study of Poisson states. Although the unnormalized nature of the operators may require extra care, one can still take advantage of many known results concerning unnormalized positive-semidefinite matrices [22, 35, 36, 38], as the formulas here coincide with many of them.

There are many potential generalizations and extensions. It should be possible to define the Poisson limit more generally for a tensor product of non-identical states and an infinite-dimensional $\Gamma$, in analogy with the more general Poisson limit theorems [1, 2]. The mathematical rigor of the limit may be improved through quantum stochastic calculus [25] or nonstandard analysis [26, 27]. The mathematical theory of Poisson channels may be refined further.

In terms of applications, some specialized aspects of the Poisson theory have already found success in the study of weak thermal light for optical sensing and imaging $[8,18]$, but a consolidation of the results under the umbrella of Poisson quantum information may reveal new insights. Given the importance of the classical Poisson theory in diverse areas [1, 2], the quantum Poisson theory is envisioned to see wider applications in quantum technologies beyond optics, wherever a sequence of rare objects may be encountered.

\section{Acknowledgments}

I acknowledge helpful discussions with Zdeněk Hradil, Nick Vamivakas, Vincent Tan, and Marco Tomamichel. This work is supported by the National Research Foundation (NRF) Singapore, under its Quantum Engineering Programme (Award QEP-P7). 


\section{A Poisson limit theorem}

To state the Poisson limit theorem rigorously, I first rephrase the measurement model in terms of measure theory. Consider a multi-output object-counting measurement of the $k$ th temporal mode. Let $\mathcal{Y}$ denote the set of outputs and $\mathcal{B}$ denote its $\sigma$-algebra. For example, for an ideal direct-imaging measurement, each $(x, y) \in \mathcal{Y}=\mathbb{R}^{2}$ is a possible position of the object on the image plane, while each $B \in \mathcal{B}$ is a region of the image plane. Let $X_{k} \in\{0,1\}$ be the total object number detected by the measurement. Assume

$$
\mathbb{E}\left[X_{k}=0\right]=\operatorname{tr} I_{0} \tau=1-\epsilon,
$$

where $\mathbb{E}$ denotes the expectation, [statement] is the Iverson bracket defined as

$$
[\text { statement }] \equiv \begin{cases}1, & \text { statement is true } \\ 0, & \text { otherwise }\end{cases}
$$

and $\mathbb{E}[$ statement $]$ is the probability that the statement is true. Conditioned on $X_{k}=1$, let $Y_{k} \in \mathcal{Y}$ be the output that detects the object. Assume

$$
\mathbb{E}\left[Y_{k} \in B\right]=\operatorname{tr} E(B) \tau_{1},
$$

where $E: \mathcal{B} \rightarrow \mathcal{P}\left(\mathcal{H}_{1}\right)$ is a POVM on $\mathcal{H}_{1}$. The object count in a set of outputs can then be modeled by the random measure $\mathcal{N}^{(k)}: \mathcal{B} \rightarrow\{0,1\}$, defined as

$$
\mathcal{N}^{(k)}(B) \equiv X_{k}\left[Y_{k} \in B\right] .
$$

$X_{k}$ and $Y_{k}$ can be taken as independent random variables. Integrated over $M$ temporal modes, the random measure becomes

$$
\mathcal{M}_{M}(B) \equiv \sum_{k=1}^{M} \mathcal{N}^{(k)}(B)=\sum_{k=1}^{M} X_{k}\left[Y_{k} \in B\right]=\sum_{j=1}^{L_{M}}\left[Z_{j} \in B\right]
$$

where

$$
L_{M} \equiv \sum_{k=1}^{M} X_{k}
$$

is the detected object number in total and the set $\left\{Z_{1}, \ldots, Z_{L_{M}}\right\}$ is simply a relabeling of $\left\{Y_{k} \mid X_{k}=\right.$ $1\}$, which is a set of $L_{M}$ independent and identically distributed (i.i.d.) random elements. As $X$ is Bernoulli, $L_{M}$ is binomial. In the Poisson limit, $L_{M}$ becomes Poisson, denoted by $L$, and $\mathcal{M}_{M} \rightarrow \mathcal{M}$ is called the Kac empirical point process [24].

Theorem 1 (see, for example, Ref. [24, Sec. 3.5.2]). With a Poisson L and a sequence of i.i.d. random elements $\left\{Z_{1}, Z_{2}, \ldots\right\}$ that are independent of $L$, the Kac process $\mathcal{M}: \mathcal{B} \rightarrow \mathbb{N}_{0}$, defined as

$$
\mathcal{M}(B) \equiv \sum_{j=1}^{L}\left[Z_{j} \in B\right]
$$

is a Poisson process.

The intensity measure $\Lambda: \mathcal{B} \rightarrow \mathbb{R}_{\geq 0}$ of the Poisson process is given by

$$
\Lambda(B)=\mathbb{E}[\mathcal{M}(B)]=N \operatorname{tr} E(B) \tau_{1}=\operatorname{tr} E(B) \Gamma .
$$

In the main text, the abbreviations $\mathcal{N}_{j}^{(k)}=\mathcal{N}^{(k)}\left(B_{j}\right), \mathcal{M}_{j}=\mathcal{M}\left(B_{j}\right), E_{j}=E\left(B_{j}\right)$, and $\Lambda_{j}=\Lambda\left(B_{j}\right)$ are used, where $\left\{B_{1}, B_{2}, \ldots\right\}$ is a disjoint partition of $\mathcal{Y} .\left\{\mathcal{M}_{j}\right\}$ are independent Poisson random variables by a basic property of Poisson processes, so their probability distribution is given by Eq. (2.7). 


\section{B Poisson states on Fock spaces}

$\left(\mathcal{H}_{0} \oplus \mathcal{H}_{1}\right)^{\otimes M}$ is called a toy Fock space in Refs. [25, 46]. The Poisson limit of $\rho_{M}$ on the toy Fock space should remain well defined if one is willing to adopt nonstandard analysis, taking $M$ to be an unlimited natural number and $\epsilon=N / M$ to be infinitesimal $[26,27]$. In the context of ordinary calculus, one may think of $\epsilon$ as $O(d t)$, where $d t$ is an infinitesimal interval in time or a similar continuous degree of freedom. In the Poisson channel theory, Eq. (5.12) implies that each Kraus operator $A_{\alpha 10}$ should scale as $O(\sqrt{\epsilon})=O(\sqrt{d t})$, which is why quantum stochastic calculus may be needed to define the operators rigorously in the Poisson limit.

A more standard way to deal with an infinite number of temporal modes in quantum stochastic calculus is to abandon the toy Fock space and use instead the Fock space $\mathcal{F}\left(\mathcal{L}^{2}[0,1] \otimes \mathcal{H}_{1}\right)$, where $\mathcal{F}$ is defined as $[25]$

$$
\mathcal{F}(\mathcal{H}) \equiv \bigoplus_{l=0}^{\infty} \mathcal{H}^{\otimes l}, \quad \quad \mathcal{H}^{\otimes 0}=\mathcal{H}_{0},
$$

and the $\mathcal{L}^{2}[0,1]$ space, modeling the temporal modes for one object, is defined as

$$
\begin{aligned}
\mathcal{L}^{2}[0,1] & \equiv\{f \mid f:[0,1] \rightarrow \mathbb{C},\langle f, f\rangle<\infty\}, \\
\langle f, g\rangle & \equiv \int_{0}^{1} f^{*}(t) g(t) d t .
\end{aligned}
$$

See Refs. $[25,26,46]$ for discussions of the relation between the toy Fock space and $\mathcal{F}\left(\mathcal{L}^{2}[0,1] \otimes \mathcal{H}_{1}\right)$. It is an open problem, outside the scope of this paper, how Poisson states may be expressed on $\mathcal{F}\left(\mathcal{L}^{2}[0,1] \otimes \mathcal{H}_{1}\right)$.

If the objects are collected in one place and their arrival times are ignored, one can assume a simpler Fock space

$$
\mathcal{F}\left(\mathcal{H}_{1}\right)=\bigoplus_{l=0}^{\infty} \mathcal{H}_{1}^{\otimes l}
$$

and transform $\rho_{M}$ into a state on $\mathcal{F}\left(\mathcal{H}_{1}\right)$ given by

$$
\begin{aligned}
\Pi\left(\rho_{M}\right) & =\bigoplus_{l=0}^{M} P_{L_{M}}(l) \tau_{1}^{\otimes l}, \\
P_{L_{M}}(l) & =\left(\begin{array}{c}
M \\
l
\end{array}\right) \pi_{0}^{M-l} \pi_{1}^{l},
\end{aligned}
$$

where $\Pi$ denotes an appropriate map, $L_{M}$ is the binomial random variable for the total object number, and $\tau_{1}^{\otimes 0}=\tau_{0}$ is assumed. This representation may be useful because any function of density operators that satisfies a monotonicity relation with respect to TPCP maps can be computed using $\Pi\left(\rho_{M}\right)$ instead of $\rho_{M}$, by virtue of the following proposition.

Proposition 12. $\Pi$ is TPCP. Moreover, there exists another TPCP map $\Pi^{\prime}$ such that $\Pi^{\prime}\left[\Pi\left(\rho_{M}\right)\right]=$ $\rho_{M}$

The proof is delegated to Appendix C.

In the Poisson limit, $L_{M}$ becomes Poisson, viz.,

$$
P_{L_{M}}(l) \rightarrow P_{L}(l)=\exp (-N) \frac{N^{l}}{l !},
$$

and the state on $\mathcal{F}\left(\mathcal{H}_{1}\right)$ becomes

$$
\Pi\left(\rho_{M}\right) \rightarrow \Pi(\rho)=\bigoplus_{l=0}^{\infty} P_{L}(l) \tau_{1}^{\otimes l}=\exp (-N) \bigoplus_{l=0}^{\infty} \frac{\Gamma^{\otimes l}}{l !} .
$$


$\Pi(\rho)$ has the spirit of a Kac process and may serve as a rigorous definition of a Poisson state without resorting to nonstandard analysis. For example, a Poisson process can arise directly from a measurement on $\Pi(\rho)$ as follows. Let $L$ be the object number determined by a measurement in terms of the Kraus operators $\left\{I_{0}, I_{1}, \ldots\right\}$, where $I_{l}$ is the projection operator into $\mathcal{H}_{1}^{\otimes l}$. The conditional state becomes $\tau_{1}^{\otimes L}$. If each object is then measured by the POVM $E$, the outcomes are the i.i.d. random elements $\left\{Z_{1}, \ldots, Z_{L}\right\}$. The Kac process $\mathcal{M}(B)=\sum_{j=0}^{L}\left[Z_{j} \in B\right]$ is therefore a Poisson process by Theorem 1 .

Many other results in this paper can also be rederived from Eq. (B.8). Equations (2.1) are more intuitive from the physics point of view, however, and are therefore used in the main text.

\section{Proofs of Propositions 2, 3, and 12}

Proof of Proposition 2. To derive Eq. (3.6), take similar steps to those in the proof of Proposition 1 to obtain

$$
\begin{aligned}
C_{s}\left(\rho_{M}, \rho_{M}^{\prime}\right) & =\left[C_{s}\left(\tau, \tau^{\prime}\right)\right]^{M}=\left[\sum_{l} C_{s}\left(\pi_{l} \tau_{l}, \pi_{l}^{\prime} \tau_{l}^{\prime}\right)\right]^{M} \\
& =\left[1-s \epsilon-(1-s) \epsilon^{\prime}+\epsilon^{s} \epsilon^{\prime 1-s} C_{s}\left(\tau_{1}, \tau_{1}^{\prime}\right)+O\left(\epsilon^{2}\right)\right]^{M},
\end{aligned}
$$

and then take the Poisson limit.

Note that Eq. (3.7) takes the infimum of the Poisson limit, but to prove that it is the same as the Poisson limit of the infimum, one may need to prove the uniform convergence of $C_{s}\left(\rho_{M}, \rho_{M}^{\prime}\right)$ to $C_{s}\left(\rho, \rho^{\prime}\right)$ over $0 \leq s \leq 1$, beyond the pointwise convergence just proved. This complication suggests that the Poisson limit is an intricate mathematical issue, and the Fock-space representation given by Eq. (B.8), the Poisson limit of which has already been taken, may be a better starting point for rigorous proofs. In particular, Eq. (B.8) leads directly to Eqs. (3.6) and (3.7) without any ambiguity.

Proof of Proposition 3. Consider

$$
\begin{aligned}
D\left(\rho_{M} \| \rho_{M}^{\prime}\right) & =M D\left(\tau \| \tau^{\prime}\right) \\
& =M \sum_{l} \operatorname{tr} \pi_{l} \tau_{l}\left[\ln \left(\pi_{l} \tau_{l}\right)-\ln \left(\pi_{l}^{\prime} \tau_{l}^{\prime}\right)\right] \\
& =M \operatorname{tr}(1-\epsilon) \tau_{0}\left\{\ln \left[(1-\epsilon) \tau_{0}\right]-\ln \left[\left(1-\epsilon^{\prime}\right) \tau_{0}\right]\right\}+M \operatorname{tr} \epsilon \tau_{1}\left[\ln \left(\epsilon \tau_{1}\right)-\ln \left(\epsilon^{\prime} \tau_{1}^{\prime}\right)\right] \\
& =M(1-\epsilon) \ln \frac{1-\epsilon}{1-\epsilon^{\prime}}+\operatorname{tr} \Gamma\left(\ln \Gamma-\ln \Gamma^{\prime}\right),
\end{aligned}
$$

where Eq. (C.3) has used Ref. [22, Eq. (5.97)] and Eq. (C.6) has used Ref. [22, Eq. (5.99)]. The Poisson limit of the first term in Eq. (C.6) is

$$
M(1-\epsilon) \ln \frac{1-\epsilon}{1-\epsilon^{\prime}}=(1-\epsilon) \ln \left[1+\epsilon^{\prime}-\epsilon+O\left(\epsilon^{2}\right)\right]^{M} \rightarrow N^{\prime}-N,
$$

which leads to Eq. (3.9).

Proof of Proposition 12. To prove that $\Pi$ is TPCP, I construct a physical procedure that takes $\rho_{M}$ as input and produces $\Pi\left(\rho_{M}\right)$ as output.

1. Designate the input system as system $\mathrm{A}$ and an auxiliary system with Hilbert space $\left(\mathcal{H}_{0} \oplus\right.$ $\left.\mathcal{H}_{1}\right)^{\otimes M}$ and initial state $\tau_{0}^{\otimes M}$ as system B.

2. For each $k=1, \ldots, M$ :

(a) Measure the number of objects in the $k$ th temporal mode of system A with the Kraus operators $\left\{I_{0}, I_{1}\right\}$. Let $X_{k} \in\{0,1\}$ be the random variable from the measurement.

(b) If $X_{k}=0$, do nothing. 
(c) If $X_{k}=1$, put the object detected in the $k$ th temporal mode of system A (in conditional state $\left.I_{1} \tau I_{1} /\left(\operatorname{tr} I_{1} \tau I_{1}\right)\right)$ into an empty temporal mode of system B (any mode in state $\left.\tau_{0}\right)$.

3. Discard all the empty temporal modes in system B so that its Hilbert space becomes $\mathcal{H}_{1}^{\otimes L_{M}}$, where $L_{M} \equiv \sum_{k=1}^{M} X_{k}$ is the number of detected objects.

4. Give system B as the output.

With $\rho_{M}$ as the input state, $X$ is Bernoulli, $L_{M}$ is binomial, the output state conditioned on $L_{M}$ is $\tau_{1}^{\otimes L_{M}}$, and the unconditional output state becomes Eq. (B.5).

To prove that a TPCP map giving $\Pi^{\prime}\left[\Pi\left(\rho_{M}\right)\right]=\rho_{M}$ exists, I construct a physical procedure that takes $\Pi\left(\rho_{M}\right)$ as input and gives $\rho_{M}$ as output.

1. Assume a system A with Hilbert space $\left(\mathcal{H}_{0} \oplus \mathcal{H}_{1}\right)^{\otimes M}$ and initial state $\tau_{0}^{\otimes M}$. Assume a system B with Hilbert space $\oplus_{l=0}^{M} \mathcal{H}_{1}^{\otimes l}$. For the $\Pi^{\prime}$ map, assume that system A is auxiliary and system $B$ is the input.

2. Measure the number of objects $L_{M}$ in system B with the Kraus operators $\left\{I_{0}, I_{1}, \ldots\right\}$.

3. Generate a random classical bit sequence $X=\left\{X_{1}, \ldots, X_{M}\right\} \in\{0,1\}^{M}$ with $L_{M}$ bits equal to 1 and $M-L_{M}$ bits equal to 0 . The probability of each sequence is assumed to be

$$
P_{X \mid L_{M}}(x \mid l)=\left(\begin{array}{c}
M \\
l
\end{array}\right)^{-1}\left[\sum_{k} x_{k}=l\right] .
$$

4. For each $k=1, \ldots, M$ :

(a) If $X_{k}=0$, do nothing.

(b) If $X_{k}=1$, put an object in system B into the $k$ th temporal mode of system A.

5. Give system A as the output.

With $\Pi\left(\rho_{M}\right)$ as the input state, $L_{M}$ is binomial, and the unconditional probability of each bit sequence becomes

$$
P_{X}(x)=\sum_{l=0}^{M} P_{L_{M}}(l) P_{X \mid L_{M}}(x \mid l)=\sum_{l=0}^{M} \pi_{1}^{l} \pi_{0}^{M-l}\left[\sum_{k} x_{k}=l\right]=\prod_{k=0}^{M}\left(\left[x_{k}=0\right] \pi_{0}+\left[x_{k}=1\right] \pi_{1}\right),
$$

meaning that $X$ is Bernoulli. Conditioned on $X$, the output state is

$$
\rho_{M}(X)=\bigotimes_{k=1}^{M}\left(\left[X_{k}=0\right] \tau_{0} \oplus\left[X_{k}=1\right] \tau_{1}\right)
$$

The unconditional output state is hence

$$
\mathbb{E}\left[\rho_{M}(X)\right]=\bigotimes_{k=1}^{M}\left(\pi_{0} \tau_{0} \oplus \pi_{1} \tau_{1}\right)=\rho_{M}
$$

\section{Computation of the Helstrom information for Example 1}

To compute Eqs. (3.27) and (3.28) numerically, I follow the method in Refs. [47, 48]. Express each operator in the problem as

$$
A=\sum_{j, k} \tilde{A}_{j k}\left|\psi_{j}\right\rangle\left\langle\psi_{k}\right|,
$$


where the set $\left\{\left|\psi_{j}\right\rangle\right\}$ spans $\mathcal{H}_{1}$. The set need not be orthogonal or normalized. Let $\Delta \equiv \partial \Gamma / \partial \theta$ for a scalar parameter $\theta$. Then Eq. (3.28) can be expressed as

$$
2 \tilde{\Delta}=\tilde{S} G \tilde{\Gamma}+\tilde{\Gamma} G \tilde{S},
$$

where $G_{j k}=\left\langle\psi_{j} \mid \psi_{k}\right\rangle$, while Eq. (3.27) can be expressed as

$$
K=\operatorname{tr} G \tilde{S} G \tilde{\Delta} .
$$

For Example 1, $\left|\psi_{1}\right\rangle$ and $\left|\psi_{2}\right\rangle$ are defined in Eqs. (4.8). Define also

$$
\left|\psi_{3}\right\rangle \equiv \frac{\partial\left|\psi_{1}\right\rangle}{\partial \theta}, \quad\left|\psi_{4}\right\rangle \equiv \frac{\partial\left|\psi_{2}\right\rangle}{\partial \theta} .
$$

Then

$$
\tilde{\Gamma}=N_{0}\left(\begin{array}{cccc}
1 & \gamma & 0 & 0 \\
\gamma^{*} & 1 & 0 & 0 \\
0 & 0 & 0 & 0 \\
0 & 0 & 0 & 0
\end{array}\right), \quad \tilde{\Delta}=N_{0}\left(\begin{array}{cccc}
0 & 0 & 1 & \gamma \\
0 & 0 & \gamma^{*} & 1 \\
1 & \gamma & 0 & 0 \\
\gamma^{*} & 1 & 0 & 0
\end{array}\right)
$$

With the Gaussian $\psi(x)$ given by Eq. (4.10), $G$ can also be computed analytically (with the help of the Symbolic Math Toolbox on Matlab (ver. 2020b, Mathworks)):

$$
G=\left(\begin{array}{cccc}
1 & \exp \left(-\frac{\theta^{2}}{8}\right) & 0 & -\frac{\theta}{8} \exp \left(-\frac{\theta^{2}}{8}\right) \\
\exp \left(-\frac{\theta^{2}}{8}\right) & 1 & -\frac{\theta}{8} \exp \left(-\frac{\theta^{2}}{8}\right) & 0 \\
0 & -\frac{\theta}{8} \exp \left(-\frac{\theta^{2}}{8}\right) & \frac{1}{16} & \frac{\theta^{2}-4}{64} \exp \left(-\frac{\theta^{2}}{8}\right) \\
-\frac{\theta}{8} \exp \left(-\frac{\theta^{2}}{8}\right) & 0 & \frac{\theta^{2}-4}{64} \exp \left(-\frac{\theta^{2}}{8}\right) & \frac{1}{16}
\end{array}\right) .
$$

For each $\gamma$ from -1 to 1 with step size 0.2 and each $\theta$ from 0.05 to 8 with step size 0.05 , Eq. (D.2) is solved for $\tilde{S}$ using the lyap function on Matlab and $K$ is computed using Eq. (D.3). The results are plotted in Fig. 1.

To prevent the error "The solution of this Lyapunov equation does not exist or is not unique" in using the lyap function on Matlab, a tiny positive number $\delta$ is artificially introduced to $\tilde{\Gamma}_{33}$ and $\tilde{\Gamma}_{44}$. To check that $\delta$ does not affect the results significantly, the numerical analysis is repeated with various $\delta$, and it is found that the plots do not show any perceptible change with $\delta$ ranging from $10^{-13}$ to $10^{-5}$. Figure 1 uses $\delta=10^{-13}$.

The Matlab code is reproduced below. The lbmap routine to generate a color map for colorblind viewers can be downloaded from Ref. [49].

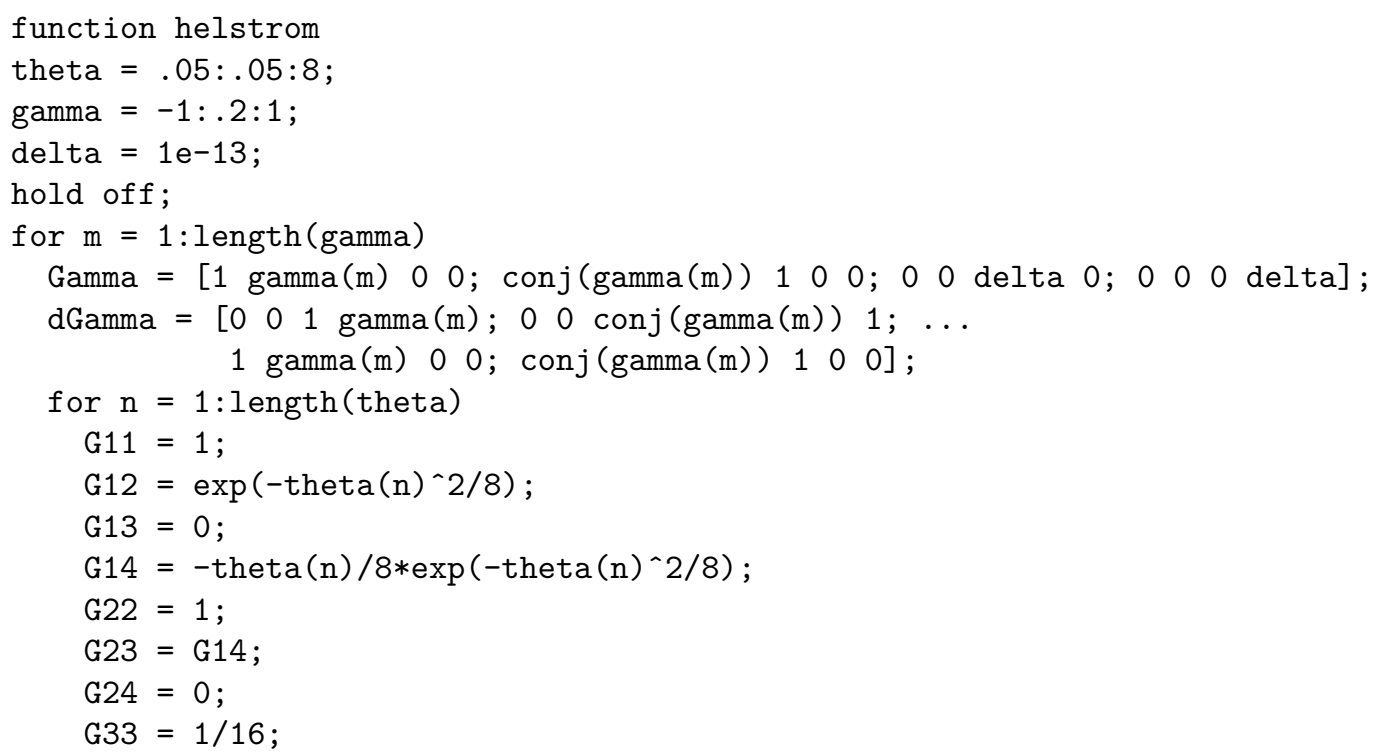




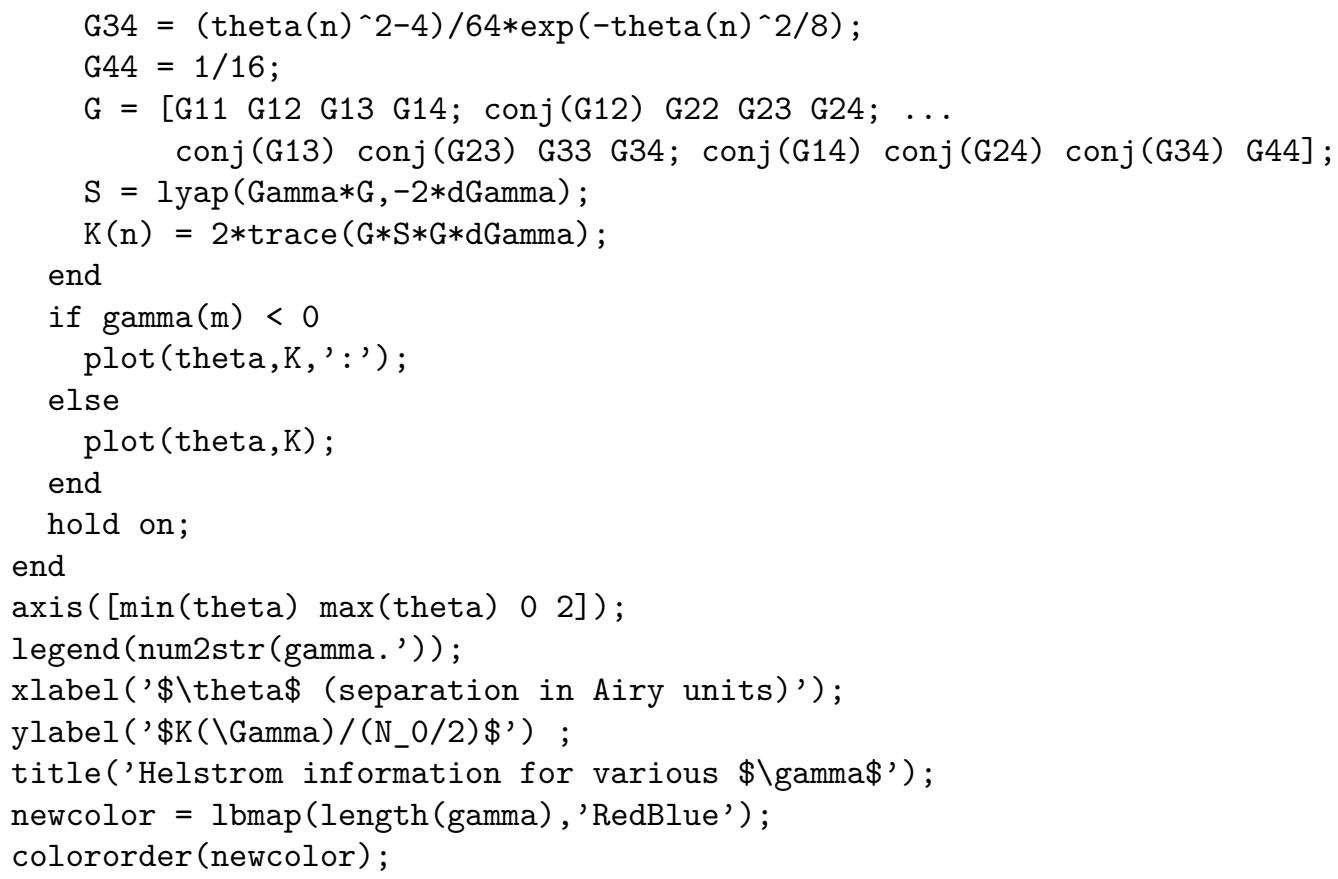

\section{References}

[1] M. Falk, J. Hüsler, and R.-D. Reiss, Laws of Small Numbers: Extremes and Rare Events, 3rd ed. (Birkhäuser, Basel, 2011).

[2] D. L. Snyder and M. I. Miller, Random Point Processes in Time and Space, 2nd ed. (SpringerVerlag, New York, 1991).

[3] C. Weedbrook, S. Pirandola, R. García-Patrón, N. J. Cerf, T. C. Ralph, J. H. Shapiro, and S. Lloyd, Rev. Mod. Phys. 84, 621 (2012).

[4] A. S. Holevo, Quantum Systems, Channels, Information, 2nd ed. (de Gruyter, Berlin, 2019).

[5] M. Tsang, Physical Review Letters 107, 270402 (2011).

[6] M. Tsang, R. Nair, and X.-M. Lu, Physical Review X 6, 031033 (2016).

[7] M. Tsang, R. Nair, and X.-M. Lu, in Proc. SPIE, Quantum and Nonlinear Optics IV, Vol. 10029 (SPIE, Bellingham, WA, 2016) p. 1002903.

[8] M. Tsang, Contemporary Physics 60, 279 (2019).

[9] W. Larson and B. E. A. Saleh, Optica 5, 1382 (2018).

[10] M. Tsang and R. Nair, Optica 6, 400 (2019).

[11] W. Larson and B. E. A. Saleh, Optica 6, 402 (2019).

[12] Z. Hradil, J. Řeháček, L. Sánchez-Soto, and B.-G. Englert, Optica 6, 1437 (2019).

[13] K. Liang, S. A. Wadood, and A. N. Vamivakas, Optica 8, 243 (2021).

[14] S. A. Wadood, K. Liang, Y. Zhou, J. Yang, M. A. Alonso, X.-F. Qian, T. Malhotra, S. M. Hashemi Rafsanjani, A. N. Jordan, R. W. Boyd, and A. N. Vamivakas, Optics Express 29, 22034 (2021).

[15] Z. Hradil, D. Koutný, and J. Řeháček, Optics Letters 46, 1728 (2021).

[16] S. De, J. Gil-Lopez, B. Brecht, C. Silberhorn, L. L. Sánchez-Soto, Z. Hradil, and J. Řeháček, Physical Review Research 3, 033082 (2021).

[17] S. Kurdzialek, arXiv:2103.12096 [physics, physics:quant-ph] (2021).

[18] C. W. Helstrom, Quantum Detection and Estimation Theory (Academic Press, New York, 1976).

[19] X.-M. Lu, H. Krovi, R. Nair, S. Guha, and J. H. Shapiro, npj Quantum Information 4, 64 (2018).

[20] D. Gottesman, T. Jennewein, and S. Croke, Physical Review Letters 109, 070503 (2012).

[21] E. T. Khabiboulline, J. Borregaard, K. De Greve, and M. D. Lukin, Physical Review Letters 123, 070504 (2019). 
[22] J. Watrous, The Theory of Quantum Information (Cambridge University Press, Cambridge, 2018).

[23] L. Mandel and E. Wolf, Optical Coherence and Quantum Optics (Cambridge University Press, Cambridge, 1995).

[24] A. W. van der Vaart and J. Wellner, Weak Convergence and Empirical Processes: With Applications to Statistics (Springer-Verlag, New York, 1996).

[25] K. R. Parthasarathy, An Introduction to Quantum Stochastic Calculus (Birkhäuser, Basel, 1992).

[26] M. Leitz-Martini, Quantum Stochastic Calculus using Infinitesimals, Ph.D. thesis, University of Tübingen, Tübingen, Germany (2001).

[27] E. Nelson, Radically Elementary Probability Theory (Princeton University Press, Princeton, New Jersey, 1987).

[28] A. Uhlmann and B. Crell, in Entanglement and Decoherence, edited by A. Buchleitner, C. Viviescas, and M. Tiersch (Springer, Berlin, 2009) pp. 1-60.

[29] M. Hayashi, Quantum Information Theory: Mathematical Foundation, 2nd ed. (Springer, Berlin, 2017).

[30] R. Bhatia, T. Jain, and Y. Lim, Expositiones Mathematicae 37, 165 (2019).

[31] K. M. R. Audenaert, J. Calsamiglia, R. Muñoz-Tapia, E. Bagan, L. Masanes, A. Acin, and F. Verstraete, Physical Review Letters 98, 160501 (2007).

[32] M. Nussbaum and A. Szkoła, The Annals of Statistics 37, 1040 (2009).

[33] A. Wehrl, Reviews of Modern Physics 50, 221 (1978).

[34] G. Lindblad, Communications in Mathematical Physics 33, 305 (1973).

[35] I. S. Dhillon and J. A. Tropp, SIAM Journal on Matrix Analysis and Applications 29, 1120 (2007).

[36] S.-i. Amari, Information Geometry and Its Applications (Springer Japan, Tokyo, 2016).

[37] T. Kailath, IEEE Transactions on Communication Technology 15, 52 (1967).

[38] A. Cichocki and S.-i. Amari, Entropy 12, 1532 (2010).

[39] M. Tsang, Physical Review A 99, 012305 (2019).

[40] R. Nair and M. Tsang, Physical Review Letters 117, 190801 (2016).

[41] C. Lupo and S. Pirandola, Physical Review Letters 117, 190802 (2016).

[42] J. H. Eberly, American Journal of Physics 40, 1374 (1972).

[43] Y. L. Len, C. Datta, M. Parniak, and K. Banaszek, International Journal of Quantum Information 18, 1941015 (2020).

[44] C. Lupo, Physical Review A 101, 022323 (2020).

[45] C. Oh, S. Zhou, Y. Wong, and L. Jiang, Physical Review Letters 126, 120502 (2021).

[46] P. A. Meyer, Quantum Probability for Probabilists, 2nd ed. (Springer-Verlag, Berlin Heidelberg, 1995).

[47] M. G. Genoni and T. Tufarelli, Journal of Physics A: Mathematical and Theoretical 52, 434002 (2019).

[48] L. Peng and X.-M. Lu, Physical Review A 103, 042601 (2021).

[49] R. Bemis, "Light Bartlein Color Maps (https://www.mathworks.com/matlabcentral/ fileexchange/17555-light-bartlein-color-maps)," MATLAB Central File Exchange (online) (2016), retrieved May 30, 2021. 